\title{
MALIGNANT OTITIS EXTERNA
}

\author{
Jyoti P. Dabholkar', Amit Sheth ${ }^{2}$
}

\begin{abstract}
Malignant Otitis Externa, though a misnomer, definitely alarms the surgeon, the idea that it behaves and spreads like a malignancy, in elderly diabetics, and if not treated deligently, can be fatal. Though the main line of treatment is medical, surgical intervention should not be deferred if indicated. We are presenting one such case where we have not only saved the patient's life but also controlled the pain adequately.
\end{abstract}

\section{INTRODUCTION}

Malignant otitis externa is a fulminant skull base osteomyelitis that appears to follow a minor episode of external otitis. All the patients included in this disease have a common base of varying degree of immunosuppresion. They are elderly, diabetics with or without haematological malignancy, with or without history of radiation. We are presenting one such case who came to us with acute facial paralysis.

\section{CASE REPORT}

GSP, a 68 year old obese, diabetic male presented to us with left ear discharge for 2 months with left lower motor neuron type of facial palsy since 20 days. The discharge was scanty, foul smelling, not blood stained and without any triggering factors. There was accompanying deep seated, severe, periauricular, dull earache.

On examination, the patient was well oriented. The right ear was normal; while the left ear external auditory canal was narrow and oedematous with a soft tissue mass covered with flakes. The tympanic membrane could not be visualised. There was left sided complete lower motor neuron facial palsy with very poor eye closure (Fig I).

The patient was admitted and an urgent C. T. scan was done showing evidence of an aggresive infection involving the left external auditory canal, middle ear and the mastoid with obvious mastoid bone osteomyelitis. There was involvement of the third part of the facial nerve canal due to inflammation (Fig II).

In view of the history of diabetes (which was found to be uncontrolled ), and C. T. scan suspecting malignant otitis externa, the patient was started on intravenous antibiotics, Inj. ciprofloxacin (400 mg) 12 hourly, Inj. gentamycin(90 $\mathrm{mg}) 8$ hourly. The patient was serially monitored with audiograms, renal function tests and estimation of blood sugar. Meanwhile a fine needle aspiration cytology was

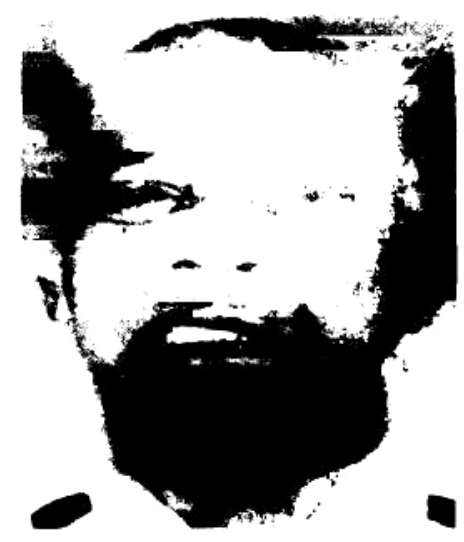

Fig. I : Clinical appearence showing complete LMN type of facial paralysis on the left.

${ }^{1}$ Professor in E.N.T, ${ }^{2}$ IInd Year Resident, B. Y. L. Ch. Hospital \& T. N. Medical College, Mumbai - 400008 


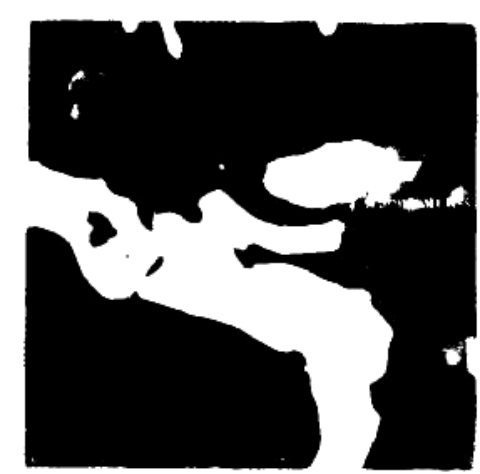

Fig. : II C T Scan

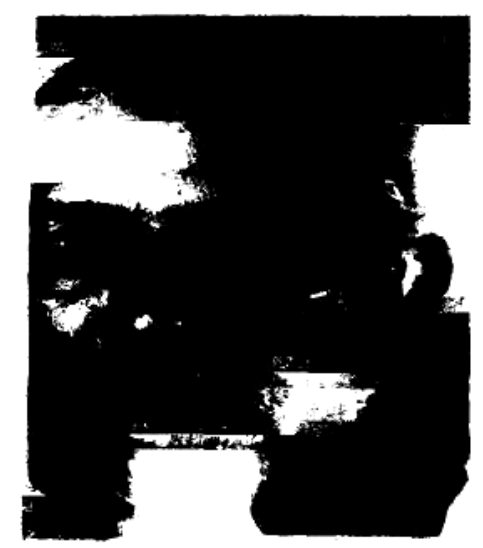

Fig : III : Post-operative appearence showing better eye closure

done of the left external auditory canal mass, which was reported as acute inflammatory lesion.

On the pursued medical line of treatment, the patient showed significant improvement in pain, but Facial Nerve function recovery was very slow and pain was persistent. Hence after two weeks of adequate antibiotic therapy and control of blood sugar, it was decided to take up the patient for mastoid exploration with facial nerve decompression.

The mastoid was exposed and was found to be pneumatic and filled with necrotic material which was removed by a simple mastoidectomy procedure. The external auditory canal was filled with epithelial debries, forming the mass seen on examination (Keratosis obturans) which was removed. The tympanic membrane was normal. A posterior tympanotomy was done and the facial nerve canal seen in its full extent. The facial nerve did not appear to be dehiscent and hence decompression of the same was not attempted.

Post-operatively, the same antibiotics were continued for two more weeks during which the pain disappeared and the facial nerve also showed significant improvement in terms of eye clousure (Fig. III).After four weeks of intravenous ciprofloxacin and gentamycin, patient was started on oral amoxycillin(500 mg) TDS and also passive facial exercises along with facial nerve galvanic stimulation was continued.

\section{DISCUSSION}

Malignant otitis externa has decreased in recent times but the incidence is still not low enough to make it a rare entity. A high rate of clinical suspicion, especially in the elderly uncontrolled diabetics with a h/o severe deep seated earache and aided with a good C. T. scan, usually helps in the diagnosis of this fulminant disease (1).

The treatment initially consists of controlling the blood sugar vigorously and also appropriate antibiotics (to which pseudomonas aeruginosa is sensitive) should be started along with a good anti-inflammatory drug. The antibiotic of choice is ciprofloxacin in high doses $(2,3)$.

Surgery, in the form of debridement of the mastoid, is reserved only for those patients where complications like facial nerve palsy as in our case or poor response to medical treatment occurs(2).

\section{ACKNOWLEDGEMENT}

Our sincere thanks to Dr. N. Kshirsagar, our Dean, for granting us the permission for publishing this paper.

\section{REFERENCES}

1. Radiology 174, 391-394.

2. Scott Brown, Sixth, ed. VOL. 3/6/17.

3. Laryngoscope, 101, 821-824.

\section{Address of Correspondent:}

Dr. Jyoti P. Dabholkar, 10, Sudha, 20 Nepean Sea Road, Mumbai 400036. 